\title{
An Autonomous Earth Observing Sensorweb
}

\author{
Steve Chien, Ben Cichy, Ashley Davies, \\ Daniel Tran, Gregg Rabideau, \\ Rebecca Castano, Rob Sherwood, \\ Son Nghiem \\ Jet Propulsion Laborator \\ California Institute of Technology \\ Ronald Greeley, Thomas Doggett \\ Arizona State University \\ Victor Baker, James Dohm, Felipe Ip \\ University of Arizona
}

\begin{abstract}
We describe a network of sensors linked by software and the internet to an autonomous satellite observation response capability. This sensor network is designed with a flexible, modular, architecture to facilitate expansion in sensors, customization of trigger conditions, and customization of responses.

This system has been used to implement a global surveillance program of multiple science phenomena including: volcanoes, flooding, cryosphere events, and atmospheric phenomena. In this paper we describe the importance of the Earth observing sensorweb application as well as overall architecture for the network
\end{abstract}

Keywords: Remote Sensing, Spacecraft Autonomy, Artificial Intelligence.

\section{Introduction}

Near Antarctica, in a remote area of the South Atlantic Ocean, a volcano rumbles. Following a few minor tremors, fresh lava suddenly breaks to the surface, flowing out of an existing vent. In years past, such an episode might have passed unnoticed or have come to light only days or weeks after the event. Now, thanks to the Earth observing sensorweb developed by the Jet Propulsion Laboratory and Goddard Space Flight Center, volcanologists around the world will have key science data about eruptions within hours.

Although the South Sandwich Islands are uninhabited, NASA's Terra and Aqua satellites fly overhead four times per day, skimming past at 7.5 kilometers per second and an altitude of 705 kilometers. Each spacecraft carries a Moderate Resolution Imaging Spectroradiometer (MODIS) instrument, which acquires resolution data of 250 to 1,000

\author{
Dan Mandl, Stuart Frye ${ }^{1}$, Seth Shulman ${ }^{2}$, \\ Stephen Ungar, Thomas Brakke, Lawrence Ong, \\ Jacques Descloitres, Jeremy Jones, \\ Sandy Grosvenor, \\ Goddard Space Flight Center \\ Rob Wright, Luke Flynn, Andy Harris \\ University of Hawaii
Robert Brakenridge, Sebastien Cacquard Dartmouth Flood Observatory

meters/pixel about the islands as part of a 2,700-kilometerwide swath of imagery.

Streamed to Goddard Space Flight Center (GSFC), the data is processed at the Distributed Active Archive Center (DAAC) where MODVOLC (MODIS VOLCano Thermal Alert System) algorithms developed at the University of Hawaii (http://modis.higp.hawaii.edu) automatically detect the volcanic activity's hot-spot signature within hours of data acquisition.

Software, monitoring the MODVOLC Web site, matches this new alert with a previously specified science team interest in volcanoes in this region, generating an observation request to the Earth Observing-1 (EO-1) ground system. Based on the request's priority, the ground system uplinks the observation request to the EO-1 spacecraft. Onboard software evaluates the request, orients the spacecraft, and operates the science instruments to acquire high - resolution (up to $10 \mathrm{~m} / \mathrm{pixel}$ ) images with hyperspectral (220 or more bands) data for science analysis. Onboard, EO-1 processes this data to extract the volcanic eruption's signature, downlinking this vital information within hours.

--The EO-1 sensorweb has demonstrated this and similar scenarios since its first operations in August 2003.

A wide range of operations satellite/platforms make their data freely available (e.g. broadcast or internet) in a rapid fashion (tens of minutes to several hours from acquisition). For example, data from MODIS flying on Terra and Aqua are available via Direct Broadcast in near real-time for regional coverage and 3-6 hours from acquisition from the GSFC Distributed Active Archive Center (DAAC). This data provides regional or global coverage with a wide range of sensing capabilities. For example, MODIS covers the globe roughly 4 times daily (two day and two night overflights). The QuikSCAT instrument flying on the SeaWinds spacecraft covers the majority of the globe daily. 
Unfortunately, these global coverage instruments do not provide the high resolution data desirable for many science applications. The above instruments range in resolution from MODIS with $250 \mathrm{~m}-1 \mathrm{~km}$ resolution to $1 \mathrm{~km}$ and above for the other instruments. While ideally high resolution data would be available continuously with global coverage, typically high resolution assets can image only limited swathes of the Earth - thus making them highly constrained and in high demand.

In this paper we describe initial efforts to network sensors and science event recognizers/trackers with an automated response system to form a sensorweb, defined as follows.

Sensorweb $A$ networked set of instruments in which information from one or more sensors is automatically used to reconfigure the remainder of the sensors

Specifically, in our application, we use low resolution, high coverage sensors to trigger observations by high resolution instruments. Note that there are many other rationales to network sensors into a sensorweb. For example automated response might enable subsequent observations using complementary instruments such as imaging radar, infrared, visible, etc. Or automated response might be used to apply more assets to increase the frequency of observation to improve the temporal resolution of available data.

In the remainder of the paper we first describe our preliminary sensorweb efforts to track

- Volcanoes

- Floods

- Wildfires

- Cryosphere events (snowfall and melt, lake freezing and thawing, sea ice formation and breakup)

We also describe ongoing efforts to expand the sensorweb to other remote sensing and in-situ assets.

\section{Sensorweb Scenario}

The EO-1 sensorweb architecture consists of a number of components which operate in the following sequence of steps.

1. Assetl acquires data (usually global coverage at low resolution)

2. Data from Asset1 is downlinked

3. This data is automatically processed to detect science events

4. Science event detections are forwarded to a re-tasking system. This system generates an observation request which is forwarded to an automated planning system.

5. This automated planning system then generates a command sequence to acquire the new observation.
6. This new command sequence is uplinked to Asset2 which then acquires the high resolution data.

7. This data is then downlinked, processed, and forwarded to the interested science team.

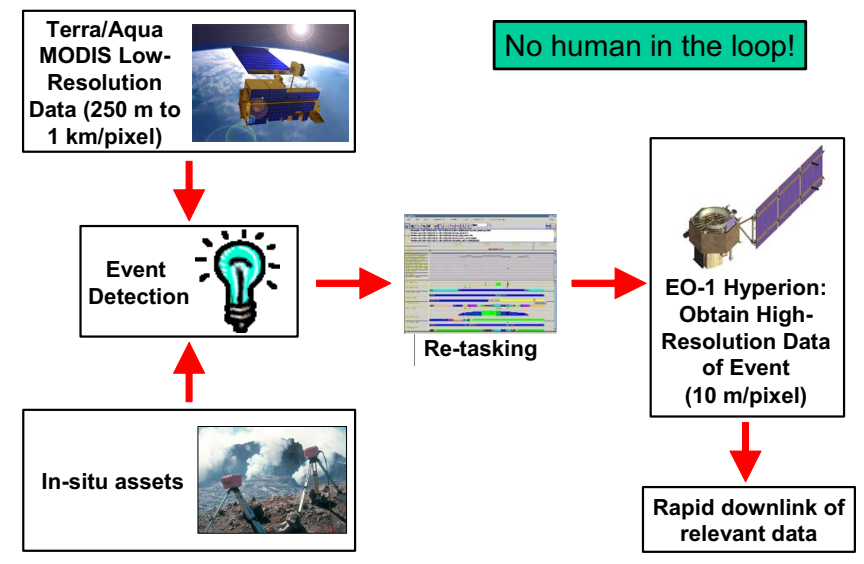

Figure 1: Sensorweb Detection and Response Architecture

In our operational system thus far, Asset2 has been the Earth Observing-1 spacecraft (EO-1). EO-1 is the first satellite in NASA's New Millennium Program Earth Observing series. The primary focus of EO- 1 is to develop and test a set of advanced technology land imaging instruments.

EO-1 [10] was launched from Vandenberg Air Force Base in 2000. Its orbit allows for 16-day repeat tracks, with 3 over flights per 16-day cycle at a less than 10-degree change in viewing angle. Because EO-1 is in a near polar orbit, polar targets can be viewed more frequently.

EO-1 has two principal science instruments, the Advanced Land Imager (ALI) and the Hyperion hyper spectral instrument. The ALI is a multi-spectral imager with $10 \mathrm{~m} /$ pixel pan-band resolution and 9 spectral bands from 0.433 to $2.35 \mu \mathrm{m}$ with $30 \mathrm{~m} /$ pixel resolution. ALI images a $37 \mathrm{~km}$ wide swath. The Hyperion is a highresolution imager capable of resolving 220 spectral bands (from 0.4 to $2.5 \mu \mathrm{m}$ ) with a $30 \mathrm{~m} /$ pixel spatial resolution. The instrument images a $7.5 \mathrm{~km}$ by $42 \mathrm{~km}$ land area per image and provides detailed spectral mapping across all 220 channels with high radiometric accuracy.

In the following sections of the paper we describe:

1. The sensorweb architecture

2. The principal science detectors that have been implemented and ongoing efforts to extend the sensorweb to additional orbital and ground-based assets

3. The system architecture design goals and lessons learned.

\section{EO-1 Sensorweb Architecture}

The automated retasking element of the sensorweb consists of several components working together as follows. 
1. Science tracking systems for each of the science disciplines automatically acquire and process satellite and ground network data to track science phenomena of interest. These science tracking systems publish their data automatically to the internet in their own format. In some cases this is via the http or ftp protocol; in others, via email subscription and alert protocols.

2. Science agents either poll these sites (http or ftp) to pull science data or simply receive email notifications of ongoing science events. These science agents then produce «science event notifications » in a standard XML format which are then logged into a « science event » database.

3. The science event manager processes these science event notifications and matches them up with « science campaigns". When a match occurs, an observation request is generated.

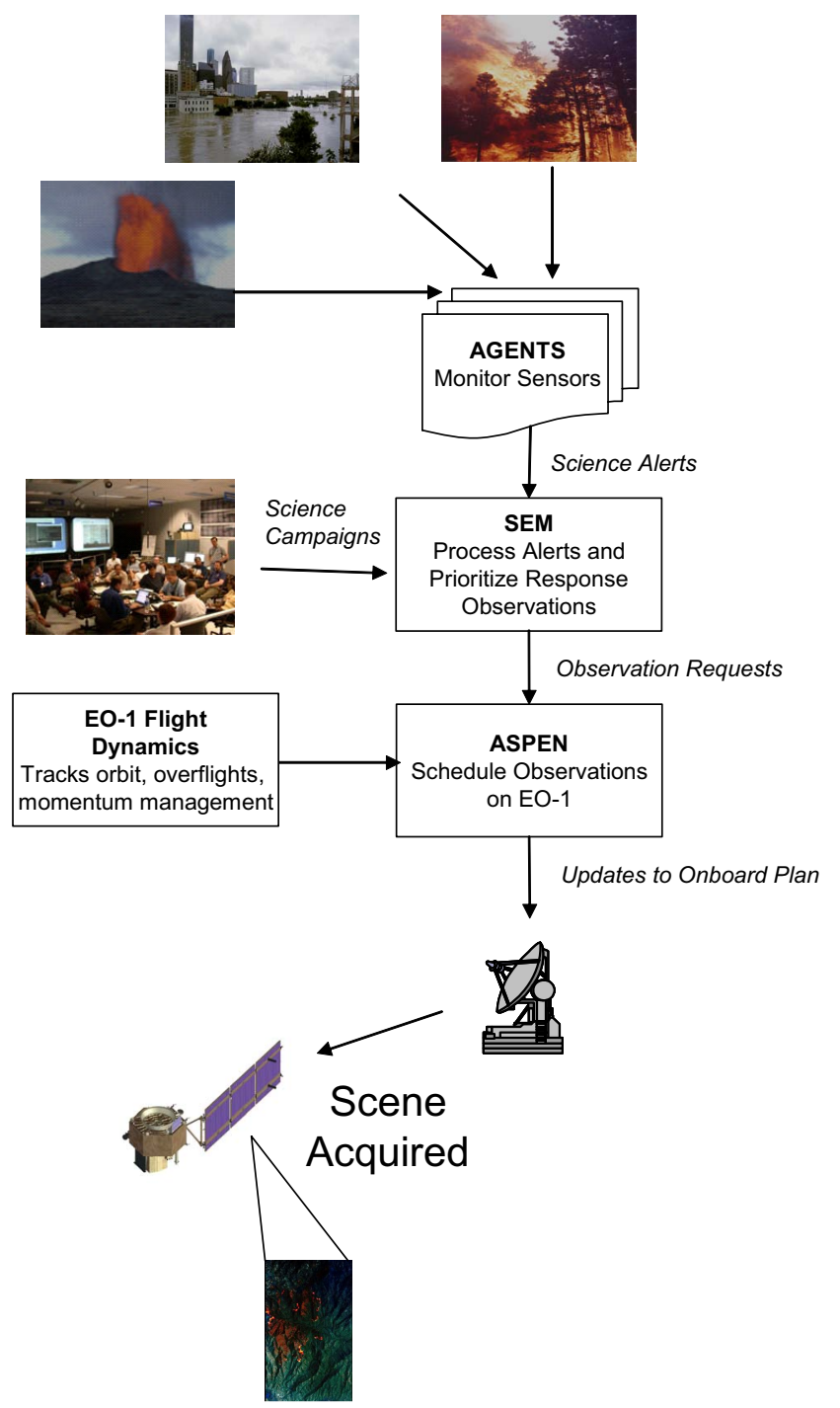

Figure 2: Sensorweb Response
4. These observation requests are processed by the ASPEN automated mission planning system. ASPEN integrates these requests and schedules observations according to priorities and mission constraints.

5. For observations that are feasible, an observation request is uplinked to the spacecraft.

6. Onboard EO-1, the Autonomous Sciencecraft software [4] will accommodate the observation request if feasible. In some cases onboard software may have additional knowledge of spacecraft resources or may have triggered additional observations so several uplinked requests may not be feasible.

7. Later, the science data is downlinked, processed, and delivered to the requesting scientist.

\subsection{Science Agents}

The science agents encapsulate sensor and science, tracking specific information by producing a generic XML alert for each "science event" tracked. The flexibility enabled by these modules has allowed us to easily integrate with a large number of science tracking systems despite each science tracking system having its own unique data and reporting format. These formats have ranged from near raw instrument data, to alerts in text format, to periodic updates, to a wide range of text formats. The posting methods have included http, https, ftp, and email. Below we list the science tracking systems integrated into our system.

\begin{tabular}{|l|l|l|}
\hline Discipline & Source & Detector \\
\hline Volcanos & MODIS (Terra Acqua) & MODVOLC, U Hawaii \\
\hline & GOES & GOESVolc \\
\hline & POES & AVHRR - Volcano \\
\hline & Air Force Weather Advisory & Volcanic Ash Alerts \\
\hline & International FAA & Volcanic Ash Advisories \\
\hline & Tungurahua, Reventador & $\begin{array}{l}\text { In-situ instruments, Harvard, } \\
\text { UNH }\end{array}$ \\
\hline Floods & Hawaiian Volcano Observatory, & Sensor alerts \\
\hline & Rabaul Volcano Observatory, ... & Dartmouth Flood Observatory \\
\hline & QuikSCAT & Dartmouth Flood Observatory \\
\hline Cryosphere & MODIS & Dartmouth Flood Observatory \\
\hline & AMSR & Snow/lce, JPLNNghiem \\
\hline Forest fires & QuikSCAT (Nghiem) & UW Dept. Limnology \\
\hline Dust Storms & Wisconsin Lake Buoys & $\begin{array}{l}\text { RAPIDFIRE, U. MD, MODIS } \\
\text { Rapid Response }\end{array}$ \\
\hline & MODIS (Terra, Acqua) & $\begin{array}{l}\text { Naval research Laboratory, } \\
\text { Monterey }\end{array}$ \\
\hline
\end{tabular}

Figure 3: Science Alert Systems

\subsection{Science Event Manager and Science Campaigns}

The science event manager enables scientists to specify mappings from science events to observation requests. It enables them to track recency and count of events and perform logical processing. It also enables them to track based on target names or locations, and other event specific parameters (for example, some tracking systems produce a confidence measure). As an example, a volcanologist 
might specify for the Kilauea site that several tracking systems would need to report activity with high confidence before an observation is requested. This is because Kilauea is quite often active. On the other hand, even a single low confidence activity notification might trigger observation of Piton de la Fournaise or other less active sites.

\subsection{Automated Observation Planning}

To automate mission planning we use the ASPEN/CASPER planning \& scheduling system [5] $]^{1}$. ASPEN represents mission constraints in a declarative format and searches possible mission plans for a plan that satisfies many observation requests while respecting priorities, and also obeys mission operations constraints. ASPEN has been used in a wide range of space mission applications including spacecraft operations scheduling, rover planning, and ground communications station automation. Figure 4 shows the graphical user interface of an EO-1 operations plan.

Figure 4: EO-1 Observation Plan as displayed by the

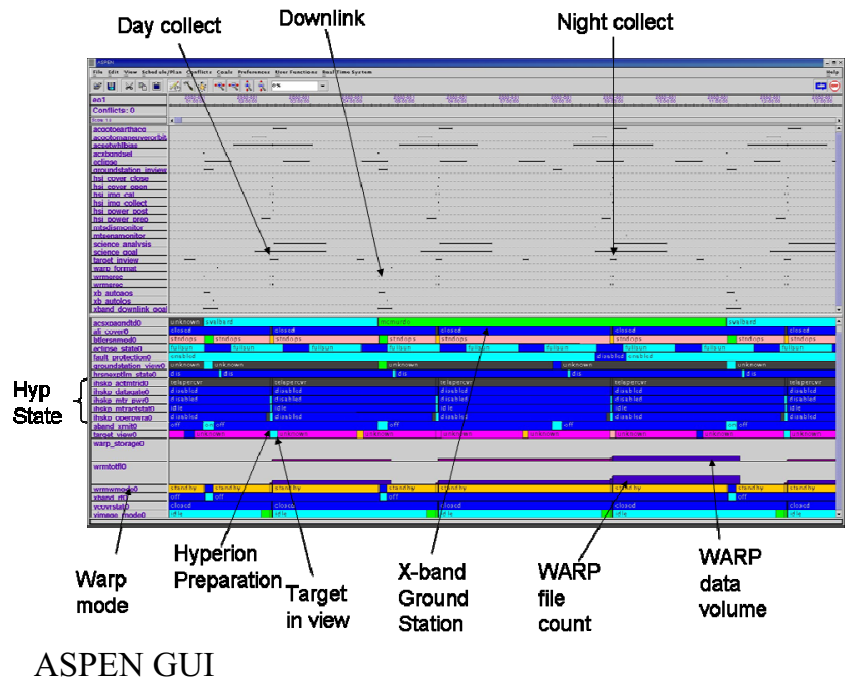

\subsection{Science Data Access}

One of the design goals of the sensorweb project has been to provide scientists easy access to multiple data sources on a single science event (such as a volcanic eruption, forest fire, etc). Unfortunately, the data access portal for the sensorweb project is still under construction.

As a related goal the sensorweb effort was to enable easy tracking of the spacecraft operations. This would enable scientists to understand the images the spacecraft had acquired and allow them to view where science products are in request, acquisition, downlink, and processing phases.

\subsection{Sensorweb Architecture Design Considerations}

\footnotetext{
${ }^{1}$ ASPEN is the ground, batch planner; CASPER is the embedded, flight planner. Both share the same core planning engine.
}

\section{and Lessons Learned}

When the sensorweb (SWB) was designed, several of the underlying systems already existed. Thus, in our design individual systems were designed or adapted to conform to logical abstract interfaces. For example, because design and deployment of a single science event tracking system might require millions of dollars, we wanted to leverage the wide range of existing science event tracking systems. This led to the creation of the science agent modules.

However, an appropriate means for assimilating event information and cross referencing it with a scientist's intent did not exist. This led to the science event manager and campaign builder development.

While EO-1 had an existing ground operations system, it did not easily support the level of automation desired for the SWB. Consequently, ASPEN was integrated into the ground operations system, requiring interfacing to EO-1 flight dynamics software and other ground support tools.

The SWB architecture was designed with several goals in mind. We review the design goals, how the design was to achieve these goals, and evaluate how well the sensorweb has achieved these goals thus far.

1. Goal: SWB should enable easy access to a wide range of existing science tracking systems. Design: Utilize a very flexible architecture with customizable science agents to allow interfacing to a wide range of science tracking systems using a range of various communications protocols.

Evaluation: The SWB has been interfaced to numerous science tracking systems with minimal effort. These interfaces have been reliable and have survived evolution and updates to the underlying tracking systems.

2. Goal: SWB should enable easy construction and modifications of science campaigns so that scientists can precisely specify their priorities and areas of interest.

Design: The science event manager allows scientists to specify science campaigns using a graphical user interface. Science campaigns can use a range of logical, threshold, and count fields to influence their campaigns and also specify the desired action as result of the event.

Evaluation: Scientists have not specified complex campaigns and tend to not modify their campaigns frequently. Even the best of interfaces does not compare to an intelligent human. In evaluating the desired action of science campaign, we currently support acquiring multiple collects over time, given a single science event. Initial work has also begun in evaluating and generating onboard science scenarios using the Autonomous Sciencecraft software onboard EO-1. (See related work).

3. Goal: The SWB should enable complete "lights out" automation so as to make rapid response imaging using EO-1 the norm rather than the exception. 
Design: Each of the steps involved in the SWB architecture was designed to enable complete automation. The mission planning step, supported by ASPEN, is the most radical automation step, replacing manual observation scheduling.

Evaluation: The overall automation flow has worked well, without significant anomalies. At this time, the sensorweb is completely automated, requiring no human intervention in processing science events, and determining the viability of observations requests. When observation requests are viable, they are automatically uplinked to EO-1 24/7 during scheduled ground contacts. However, the high demand for EO-1 imagery and the relative low priority of many of the sensorweb observations, with respect to the other observations, has reduced the number of achieved sensorweb images to 5-10 per week.

4. Goal: The SWB should enable scientists to track the spacecraft operations to view events and requests as they become spacecraft activities which then produce data, downlinked and processed.

Design: All of the sensorweb modules were designed with database enabled tracking capabilities linked to the internet via web pages.

Evaluation: Tracking pages have required significant background knowledge to interpret and thus have not been widely used by scientists. Efforts are underway to update the pages to make them more understandable.

5. Goal: The SWB should enable scientists to access a wide range of data associated with a science event, indexed by the science event not data source. Design: the SWB data access portal is designed to link in the many data sources and enable access from a single point of entry.

Evaluation: Implementation of the data portal is in its initial stages. Though all the data acquired is immediately accessible via the internet shortly after downlink, each observation has yet to be indexed to the triggering science event.

\section{Sensorweb Examples}

\subsection{The Wildfire Sensorweb}

We have demonstrated the sensorweb concept using the MODIS active fire mapping system. Both the Terra and Aqua spacecrafts carry the MODIS instrument, providing morning, afternoon, and two night overflights of each location on the globe per day (cover near the poles is even more frequent). The active fire mapping system uses data from the GSFC Distributed Active Archive Center (DAAC), specifically the data with the predicted orbital ephemeris which is approximately 3-6 hours from acquisition.
The active fire mapping algorithm [15] detects hotspots using MODIS thermal bands with absolute thresholds:

- $\quad \mathrm{T} 4>360 \mathrm{~K}, 330 \mathrm{~K}$ (night)

- $\quad \mathrm{T} 4>330 \mathrm{~K}, 315 \mathrm{~K}$ (night) and T4-T11>25K, 10K (night)

It also uses a relative threshold algorithm which requires 6 nearby pixels in an up to $21 \times 21$ square that are cloud, smoke, water, and fire free. This triggers if the thermal reading is 3 standard deviations above the surrounding area.

- $\quad \mathrm{T} 4>\operatorname{mean}(\mathrm{T} 4)+3 \operatorname{stddev}(\mathrm{T} 4)$ and $\mathrm{T} 4$ - T11 > median(T4-T11)+3stddev(T4-T11)

Figure 5 shows the active fire map from October 2003 fires in Southern California. Figure 6 shows the context active fire map and a sensorweb trigger observation taken during this demonstration.

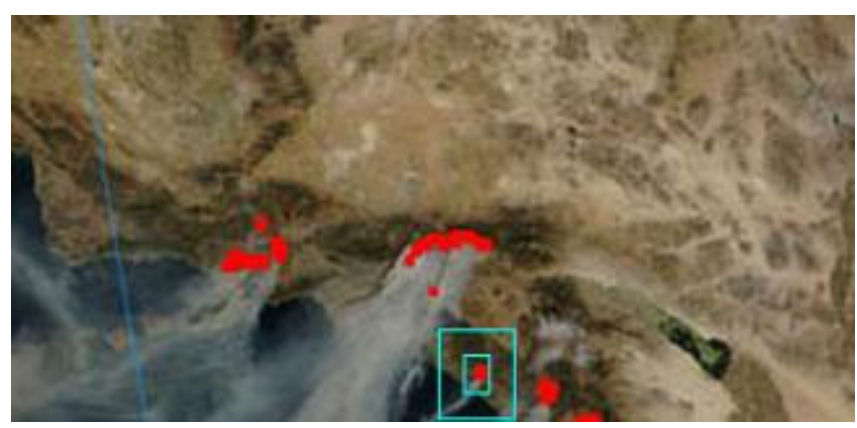

Figure 5: Active fire alerts for the recent October 2003 Southern California Fires. Red indicates active fires. The light blue box illustrates the background region used in the relative threshold detection.

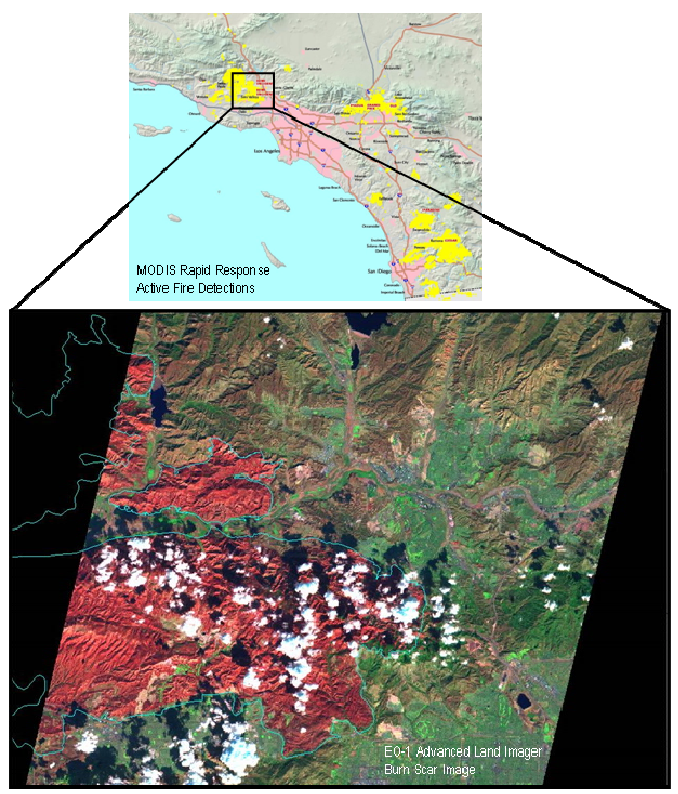


Figure 6: Sensorweb trigger images for October 2003 Southern California Fires. Above is the MODIS Active Fire Map display. Below is the EO-1 Hyperion image acquired via a sensorweb trigger of the Simi/Val Verde fire area used in Burned Area Emergency Reclamation (BAER).

\subsection{The Flood Sensorweb}

The flood sensorweb uses the Dartmouth Flood Observatory Global Active Flood Archive to identify floods in remote locations automatically based on satellite data. The DFO flood archive generates flood alerts based on both MODIS and QuikSCAT [19] satellite data. The flood sensorweb utilizes the DFO QuikSCAT atlas because it is not affected by cloud cover over flooded areas.

The DFO archive is produced by the DFO in collaboration with JPL. In this process the QuikSCAT Scatterometer data is used to assess surface water conditions $[2,18]$. Specifically the $\mathrm{VV} / \mathrm{HH}$ ratio is used to assess surface water properties of the areas in 0.25 lat/lon degree bins. The 7 day running mean is used to dampen effects of short-duration rainfall over urban areas. These data are then compared to the seasonal (90 day) average of the previous year season to screen out seasonal wetlands. The screened alerts are then published to a DFO website. More recently MODIS and AMSR-E data has been incorporated into the triggering product.

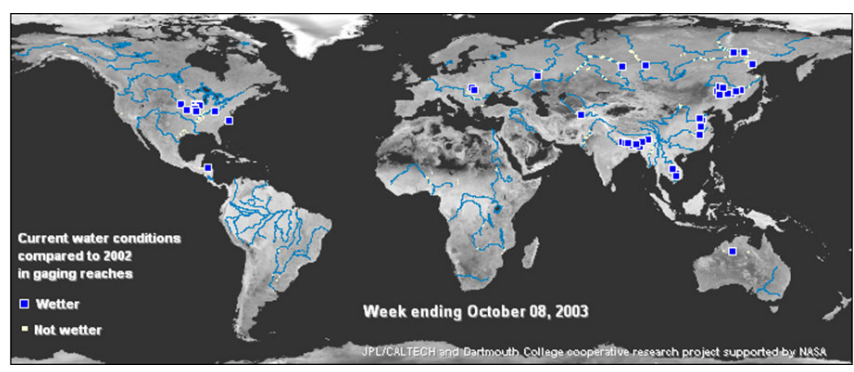

Figure 7: Dartmouth Flood Observatory Global Flood Alerts for October 2003.

In the flood sensorweb, active flooding alerts of prime locations of known scientific interest trigger EO-1 observations at gauging reaches. Gauging reaches are river locations whose topography is well understood. Flood discharge measurements at gauging reaches can be used to measure the amount of water passing through a flooded region and can be compared with remotely sensed data. The end effect of the flood sensorweb is to increase the amount of high resolution remote sensing data available on flooding events in prime locations of interest (e.g., gauging reaches) and times of interest (e.g. when active flooding occurs). Imagery from an August 2003 flood sensorweb demonstration capturing flooding in the Brahmaputra River, India, is shown in Figure 8.

\subsection{The Volcano Sensorweb}

In the volcano sensorweb, MODIS, GOES, and AVHRR sensor platforms are utilized to detect volcanic activity. These alerts are then used to trigger EO-1 observations [8]. The EO-1 Hyperion instrument is ideal for study of volcanic processes because of its great sensitivity range in the infra-red spectrum.

The GOES [13] and AVHRR alert systems provide excellent temporal resolution and rapid triggering based on thermal alerts. The GOES-based system looks for locations that are: hot, of high contrast from the surrounding area, and not visibly bright. Additionally, hits are screened for motion (to eliminate cloud reflections) and persistence (to remove instrument noise). The GOES alert can provide a web or email alert within 1 hour of data acquisition.

The MODIS alert system [22] has the advantage of high instrument sensitivity but has lower temporal resolution (MODIS generally has at least 4 overflights per day). MODVOLC derives the normalized thermal index (NTI) from MODIS raw radiance values by computing (R22 $\mathrm{R} 32) /(\mathrm{R} 22+\mathrm{R} 32)$ where $\mathrm{Ri}$ indicates the use of the radiance value from MODIS band $i$. The NTI is compared to a threshold to indicate alerts and is generally available online within 3-6 hours of acquisition.

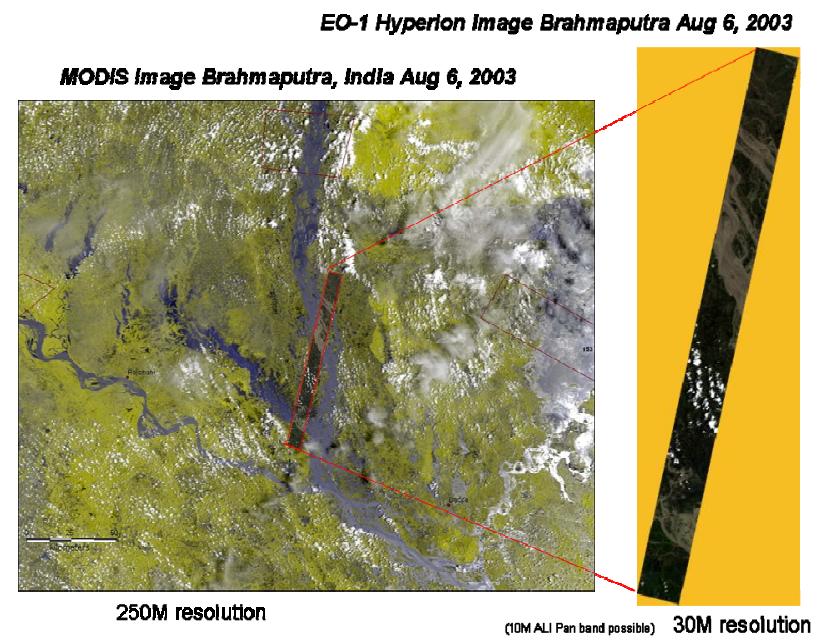

Figure 8: Examples of low-resolution MODIS imagery (left) and EO-1 imagery (right) from the flood sensorweb capturing Brahmaputra River flooding in India, August 2003.

We have also linked into in-situ sensors to monitor volcanoes. We are working with a number of teams to integrate such sensors into our sensorweb. The Hawaiian Volcano Observatory (HVO) has deployed numerous instruments on the Kilauea region in Hawaii. These instruments include tiltmeters, gas sensors, and seismic instrumentation. These sensors can provide indications that collectively point to a high-probability, near-term eruption thereby triggering a request for high-resolution, EO-1 imagery. The University of Hawaii has also deployed infra-red cameras [14] to a number of volcanic sites 
worldwide (e.g., Kilauea, Hawaii; Erte Ale, Ethiopia; Soufriere Hills, Montserrat; Colima and Popocatepetl, Mexico). These infra-red cameras can provide a groundbased detection of lava flows based on thermal signatures, thereby alerting the sensorweb.

\subsection{Cryosphere Sensorweb}

Many freeze/thaw applications are also of interest. This includes the phenomena of glacial ice breakup, sea ice breakup, melting, and freezing, lake ice freezing and thawing, and snowfall and snowmelt. Using QuikSCAT data we are tracking snow along with ice formation and melting and automatically triggering higher resolution imaging with EO-1.

In collaboration with the Center for Limnology at the University of Wisconsin at Madison, we have linked into data streams from the Trout Lake station to use temperature data to trigger imaging of the sites to capture transient freezing and thawing processes.

\subsection{Other Sensorwebs}

On average, $60 \%$ of the images acquired by EO- 1 contain cloud coverage of the science target. For some scientists, no useful science data can be obtained from these observations, requiring additional acquisitions of the target for a cloud free image. In order to reduce the number of cloud covered images, the EO-1 sensorweb network has been integrated with the Earth Phenomena Observing System (EPOS) [1] developed by Draper Laboratory. EPOS incorporates cloud data provided by the Air Force Weather Agency's (AFWA) Stochastic Cloud Forecast Model, which contains estimated cloud densities up to 84 hour forecast. In a given overflight of EO-1, EPOS receives a set of candidate targets that are requested by scientists. 12 hours prior to the target acquisition time, EPOS estimates which observations is least likely to be cloudy. This observation is then automatically uplinked to EO-1. EPOS has been operating successfully in the sensorweb since August of 2005.

Outreach work has also been done with the University of Maryland, Baltimore County (UMBC). A software agent was developed to monitor the results of sensor testing undergraduate students were performing with miniature rovers. When a science alert was issued, it was picked up by the sensorweb, processed, and an observation request was issued to EO-1 to acquire subsequent images of the UMBC campus.

\section{Ongoing Extensions and Extra-terrestrial Applications}

Terrestrial dust storms are of significant science interest and can be detected using several sensors including GOES, AVHRR, and MODIS [17]. These storms can become quite large (100s of kms long) and are of interest because of dust transport and aviation impact. A dust storm sensorweb would utilize low resolution assets to track large-scale dust storms and autonomously direct high resolution assets such as EO-1 to acquire more detailed data. Such data would improve scientific understanding of dust initiation and transport phenomena.

Figure 9 shows a large dust storm in the Persian Gulf as imaged by MODIS in November 2003. Dust storms can also be detected by ground-based instrumentation, such as operated by the U.S. Department of Agriculture in the U.S. Southwest and the Peoples Republic of China network of sites in the Gobi Desert. Detection and tracking of dust storms is also of considerable interest on Mars where such storms can grow to cover the entire planet.

The sensorweb concept is directly applicable to deep space science applications, sun-earth connection science, and astrophysics applications [6]. For example, on Mars surface instruments could detect and/or track active, transient atmospheric and geologic processes such as dust storms. Alternatively, sun-pointed instruments could detect Coronal Mass Ejections (CMEs) and alert Earth orbiting magnetospheric instruments (e.g. IMAGE, MMC, MMS, etc) to reconfigure to maximize science data.

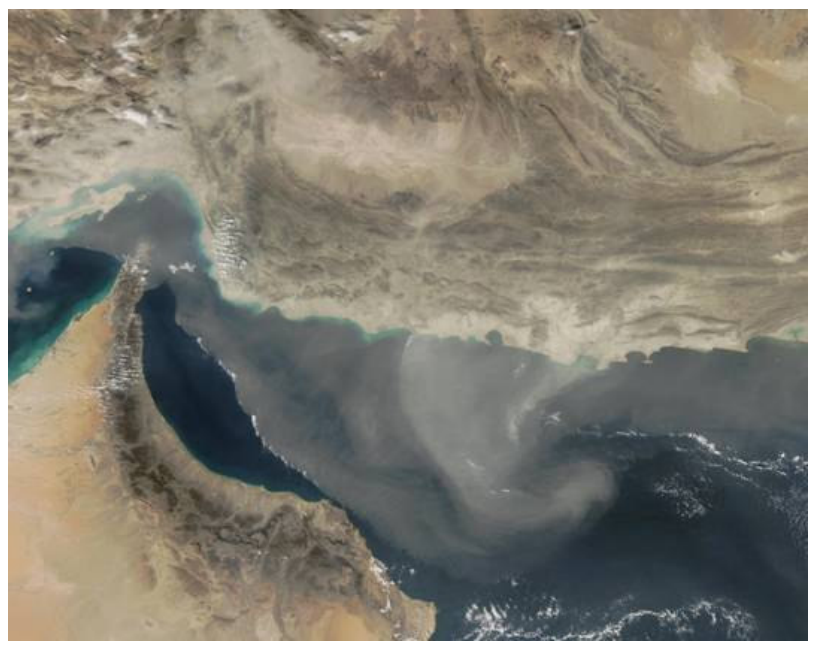

Figure 9: Dust Storm in the Persian Gulf as captured by MODIS November 2003.

\section{Related Work \& Summary}

There has been considerable effort devoted towards closed loop science for rovers at NASA Ames [12], JPL [3], and Carnegie Mellon University [21]. These efforts have some similarity in that they have science, execution, and in some cases mission planning elements. However, because surface operations (e.g. rover) are very different from orbital operations, their focus is on integration with rover path planning and localization, reliable traverse, etc., whereas our efforts focus on reliable registration of remote sensed data, interaction with orbital mechanics, and multiple platforms. The MISUS system [9] also describes a closed-

loop multi-rover autonomous science architecture. 
One closely related effort is led by Keith Golden [11] at NASA Ames to enable real-time processing of Earth Science data such as weather data. However, this work focuses on the data processing and information gathering aspect of the problem, and thus is complementary to our sensorweb work which focuses on the operations aspect of the problem. Indeed, we have discussed with Golden the possibility of a joint sensorweb information gathering demonstration.

The Autonomous Sciencecraft Experiment on EO-1 [4] demonstrates an integrated autonomous mission using onboard science analysis, replanning, and robust execution. The ASE performs intelligent science data selection and autonomous retargeting. ASE represents a single spacecraft onboard autonomous capability. In contrast the sensorweb uses multiple assets in concert.

This paper has described ongoing work to link together automated science event tracking system with an autonomous response capability based on automated planning technology. Demonstration of these sensorweb capabilities will enable fast responding science campaigns and increase the science return of spaceborne assets.

\section{References}

[1] M. Abramson et al. "Automated Coordinated Mission Planning Across Constellations". Earth Science Technology Conference (ESTC), Maryland, June 2003

[2] Brakenridge, G. R. et al. "Prospects for a Global Surface Water Observatory," EOS - Transactions of the American Geophysical Union, v86 \#19 May 2005.

[3] R. Castano et al. "Increased Mission Science Return Through In-Situ Data Prioritization", IEEE Aerospace Conference, Big Sky, Montana, March 2003.

[4] S. Chien et al. "Using Autonomy Flight Software to Improve Science Return on Earth Observing One," Journal of Aerospace Computing, Information, and Communication . April 2005.

[5] S. Chien et al., Automated Scheduling and Planning Environment (ASPEN), project home page, aspen.jpl.nasa.gov, 2003.

[6] A.G. Davies, et al. "ASC Science Report," August 2001. (downloadable from ase.jpl.nasa.gov)

[7] Davies, A. G. et al. (1999) Hypothesis-driven active data analysis of geological phenomena using semi-autonomous rovers: exploring simulations of Martian hydrothermal deposits. EOS, Trans. Amer. Geophys. Union, 80, no. 17, S210.

[8] Davies, A. G. et al. Sensor Web Enables Rapid Response to Volcanic Activity, EOS - Transactions of the American Geophysical Union, v. 87, no. 1, p1,5, January 2006.

[9] T. Estlin et al. An Integrated System for Multi-Rover Scientific Exploration, National Conference on Artificial Intelligence, Orlando, Florida, August 1999.
[10] Goddard Space Flight Center, EO-1 Mission page: http://EO1.gsfc.nasa.gov

[11] K. Golden et al. "Automating the Processing of Earth Observation Data," Intl Symposium on Artificial Intelligence, Robotics, and Automation in Space (i-SAIRAS), Nara, Japan, May 2003.

[12] V. C. Gulick et al. "Autonomous image analysis during the 1999 Marsrokhod rover field test," J. Geophysical Research, Vol.106, No. E4, pp. 7745-7764, 2001.

[13] Harris, A. et al., (2002) Web-Based Hot Spot Monitoring using GOES: What it is and How it Works, Advances in Environmental Monitoring and Modelling Vol. 1 No. 3 (2002) pp.5-36.

[14] Harris et al., Ground-based Infrared Monitoring Provides New Tool for Remote Tracking of Volcanic Activity, EOS, Vol. 84, No. 40, 7 October 2003

[15] Justice, C. et al., (2002) The MODIS fire products, Remote Sensing of Environment 83 (2002) 244-262

[16] Kratz, T., et al. "University of Wisconsin at Madison, Center for Limnology, Trout Lake Station," http://limnology.wisc.edu/tls/troutlake.html

[17] S. Miller, "A consolidated technique for enhancing desert dust storms with MODIS," Geophysical Research Letters, Vol. 30, No. 20, 2071.

[18] S. V. Nghiem et al. "Polarization Reversal over Flooded Regions and Applications to Flood Mapping with Spaceborne Scatterometers," International Geoscience and Remote Sensing Symposium, Hamburg, Germany, June 28 - July 2, 1999.

[19] S. V. Nghiem, Advanced Scatterometry for Geophysical Remote Sensing, JPL Document D-23048, 40 pages, Jet Propulsion Laboratory, California Institute of Technology, Pasadena, California, March 2001.

[20] G. Rabideau et al. "Iterative Repair Planning for Spacecraft Operations in the ASPEN System," International Symposium on Artificial Intelligence Robotics and Automation in Space, Noordwijk, The Netherlands, June 1999.

[21] T. Smith, "Science Autonomy in the Atacama," Proceedings of the International Conference on Machine Learning Workshop on Machine Learning for Autonomous Space Applications, Washington, DC, August 2003.

[22] R. Wright et al. (2002). Automated volcanic eruption detection using MODIS. Remote Sensing of Environment, 82, 135-155

\section{Acknowledgement}

Portions of this work were performed at the Jet Propulsion Laboratory, California Institute of Technology, under a contract with the National Aeronautics and Space Administration. 